\title{
ANALYSIS OF THE QUADRATIC TERM IN THE BACKSCATTERING TRANSFORMATION
}

\author{
INGRID BELTIŢĂ and ANDERS MELIN*
}

\begin{abstract}
The quadratic term in the Taylor expansion at the origin of the backscattering transformation in odd dimensions $n \geq 3$ gives rise to a symmetric bilinear operator $B_{2}$ on $C_{0}^{\infty}\left(\mathrm{R}^{n}\right) \times C_{0}^{\infty}\left(\mathrm{R}^{n}\right)$. In this paper we prove that $B_{2}$ extends to certain Sobolev spaces with weights and show that it improves both regularity and decay.
\end{abstract}

\section{Introduction and formulation of the main result}

The real part of the Fourier transform of the backscattering part of the scattering matrix associated to the Schrödinger operator $H_{v}=-\Delta+v$ in $\mathrm{R}^{n}$, where $n \geq 3$ is odd, can for $v$ in suitable function spaces be represented as a function $v+$ $\beta(v)+R$. Here $R$ is a smooth function, which is due to the negative bound states and does not appear when $v$ is small or nonnegative. The term $\beta(v)$ is entire analytic in $v$, and its Taylor series at $v=0$ starts with a quadratic term $\beta_{2}(v)$. The corresponding bilinear transformation $B_{2}$ is a singular integral operator on $C_{0}^{\infty}\left(\mathrm{R}^{n}\right) \times C_{0}^{\infty}\left(\mathrm{R}^{n}\right)$. We refer to the paper [5] in which the backscattering transform was defined in arbitrary odd dimension (see Definition 3.4 in that paper). An explicit formula for $B_{2}$ is provided by Corollary 10.7 of [5], which implies that

$$
\text { (1.1) } \begin{aligned}
& B_{2}(f, g)(x) \\
= & \iint E(y, z) f\left(x+\frac{y+z}{2}\right) g\left(x-\frac{y-z}{2}\right) \mathrm{d} y \mathrm{~d} z, \quad f, g \in C_{0}^{\infty}\left(\mathrm{R}^{n}\right) .
\end{aligned}
$$

Here $E(y, z)=4^{-1}(i \pi)^{1-n} \delta^{(n-2)}\left(|y|^{2}-|z|^{2}\right)$ is the unique fundamental solution of the ultra-hyperbolic operator $\Delta_{y}-\Delta_{z}$ such that $E(y, z)=-E(z, y)$ and $E(y, z)$ is separately rotation invariant in both variables (see Corollary 10.2 of [5]). With this notation $\beta_{2}(v)=B_{2}(v, v)$.

\footnotetext{
* The first author has been partially supported by the grant 2-CEx06-11-18/2006.

Received January 23, 2008.
} 
Since $E$ is not a function the formula (1.1) needs to be interpreted in the distribution sense. If the trilinear form $Q$ on $C_{0}^{\infty}\left(\mathrm{R}^{n}\right)$ is defined through

$$
Q(f, g, h)=\int h(x) B_{2}(f, g)(x) \mathrm{d} x, \quad f, g, h \in C_{0}^{\infty}\left(\mathrm{R}^{n}\right),
$$

this means that

$$
Q(f, g, h)=\langle E, \Phi\rangle
$$

where

$$
\Phi(y, z)=\int h(x) f\left(x+\frac{y+z}{2}\right) g\left(x-\frac{y-z}{2}\right) \mathrm{d} x .
$$

In what follows we are going to use similar notation for other integrals that have to be interpreted in the distribution sense. For definitions and basic results in distribution theory we use [3].

The expressions for $B_{2}$ and $E$ above show easily that $B_{2}$ is continuous from $C_{0}^{\infty}\left(\mathrm{R}^{n}\right) \times C_{0}^{\infty}\left(\mathrm{R}^{n}\right)$ to $C_{0}^{\infty}\left(\mathrm{R}^{n}\right)$. It commutes with translations and $|x| \leq$ $\sqrt{r_{1}^{2}+r_{2}^{2}}$ in the support of $B_{2}(f, g)$ if $|x| \leq r_{1}$ in $\operatorname{supp}(f)$ and $|x| \leq r_{2}$ in $\operatorname{supp}(g)$. From formulas we derive in the next section it will be clear that $B_{2}$ extends to a much larger domain than $C_{0}^{\infty} \times C_{0}^{\infty}$. In particular $B_{2}(f, g)$ is defined as a distribution when $f, g \in L_{\mathrm{cpt}}^{2}$.

It was noticed in the paper [4] that, when $v$ is compactly supported, the high order terms $\beta_{N}(v)$ of $\beta(v)$ gain smoothness that increases with $N$. This result was made more precise in the paper [1]. In particular, it was shown that $\beta(v)$ is more regular than $v$.

In this paper we focus our considerations to $B_{2}$ and establish continuity estimates in weighted Sobolev spaces. Specifically, we consider the spaces

$$
H_{(a, b)}\left(\mathrm{R}^{d}\right)=\left\{u \in \mathscr{S}^{\prime}\left(\mathrm{R}^{d}\right) ;\langle x\rangle^{a}\langle D\rangle^{b} u \in L^{2}\left(\mathrm{R}^{d}\right)\right\}
$$

where $a, b \in \mathrm{R}$ and $D=\mathrm{i}^{-1} \partial$, hence $\langle D\rangle$ is multiplication by $\langle\xi\rangle=(1+$ $\left.|\xi|^{2}\right)^{1 / 2}$ on the Fourier transform side. We prove then that for certain values of $a, b \geq 0$ it is true that $B_{2}$ extends to a bilinear operator on $H_{(a, b)}$. In fact, it happens also that there are $a, b, \bar{a}, \bar{b}$ with $a<\bar{a}, b<\bar{b}$ such that $B_{2}$ is continuous from $H_{(a, b)}\left(\mathrm{R}^{n}\right) \times H_{(a, b)}\left(\mathrm{R}^{n}\right)$ to $H_{(\bar{a}, \bar{b})}\left(\mathrm{R}^{n}\right)$. This means that $B_{2}$ in a certain sense improves decay and regularity at the same time and therefore shares some nice features with ordinary multiplication as well as convolution. There are good reasons to believe that similar properties hold for higher order terms $B_{N}$ in the expansion of the backscattering transform, and if so, this would have applications in inverse scattering.

Throughout this paper we use the notation $m=(n-3) / 2$. Our main result is the following theorem. 
TheOrem 1.1. Assume $\left(a^{\prime}, b^{\prime}, a^{\prime \prime}, b^{\prime \prime}, a, b\right) \in \mathrm{R}^{6}$ satisfies

$$
\begin{gathered}
0<a<m+1 / 2+\min \left(a^{\prime}, a^{\prime \prime}\right), \quad a \leq a^{\prime}+a^{\prime \prime}-1 / 2, \\
0 \leq b<1+\min \left(b^{\prime}, b^{\prime \prime}\right), \quad b+m \leq b^{\prime}+b^{\prime \prime} \\
a+b<1 / 2+\min \left(a^{\prime}, a^{\prime \prime}\right)+\min \left(b^{\prime}, b^{\prime \prime}\right)
\end{gathered}
$$

Then $B_{2}$ is continuous from $H_{\left(a^{\prime}, b^{\prime}\right)}\left(\mathrm{R}^{n}\right) \times H_{\left(a^{\prime \prime}, b^{\prime \prime}\right)}\left(\mathrm{R}^{n}\right)$ to $H_{(a, b)}\left(\mathrm{R}^{n}\right)$.

COROllary 1.2. Assume that

$$
0 \leq \bar{a} \leq a, \quad 0 \leq \bar{b} \leq b, \quad \bar{a}+\bar{b}<1 / 2 .
$$

Then $B_{2}$ is continuous from $H_{(1 / 2+a, m+b)} \times H_{(1 / 2+a, m+b)}$ to $H_{(1 / 2+a+\bar{a}, m+b+\bar{b})}$. In particular, $B_{2}$ is a continuous bilinear operator on $H_{(a, b)}$ when $a \geq 1 / 2$ and $b \geq m$.

In the result above the improvement on regularity of $\beta_{2}(v)=B_{2}(v, v)$ depends, in a certain sense, on the regularity of the potential. In particular, there is no gain in smoothness when $b=m$. It can also be noticed that when the dimension increases, the potential needs to be more regular. However, it does not need to have better decay, since the conditions on $a^{\prime}, a^{\prime \prime}$ in Theorem 1.1 do not become more restrictive when dimension increases. Let us mention the results in [6], where the cases of dimensions $n=2,3$ are considered. In the case $n=3$, these results imply that, for $L^{2}$ compactly supported potentials, the difference between the potential and the Born series approximation of the Fourier transform of the backscattering data belongs, modulo a smooth function, to the Sobolev space $H_{(0, \delta)}$ with $\delta<1 / 2$, and thus the regularity improves independently of the regularity of the potential. From the point of view of regularity our results are better, in the case $n=3$, only for potentials in $H_{(0, b)}$ with $b \geq 1 / 2$. However, as explained above, obtaining continuity properties for $B_{2}$, from which improvement both in regularity and decay can be derived, is the main aim here, and our technique is adapted to this aim.

The proof of Theorem 1.1 relies on a duality argument applied to the trilinear form $Q$ in (1.2). There is a simpler expression for $Q$. To see this, consider the bilinear operator

$$
A: \mathscr{S}\left(\mathrm{R}^{n}\right) \times \mathscr{S}\left(\mathrm{R}^{n}\right) \rightarrow \mathscr{S}\left(\mathrm{R}^{n+1}\right)
$$

defined through

$$
A(f, g)(x, t)=\int k_{0}(y, t) f(x-y) g(x+y) \mathrm{d} y, \quad x \in \mathrm{R}^{n}, t \in \mathrm{R} .
$$

Here $k_{0}(x, t)$ is the convolution kernel of the operator $K_{0}(t)=\sin (t|D|) /|D|$ that is, $K_{0}(t)$ is multiplication by $\sin (t|\xi|) /|\xi|$ on the Fourier transform side. 
We note here that $u(x, t)=K_{0}(t) f(x)$ is, for every $f \in C_{0}^{\infty}\left(\mathrm{R}^{n}\right)$, the unique solution in $C^{1}\left([0, \infty), L^{2}\left(\mathrm{R}^{n}\right)\right)$ to the Cauchy problem $\left(\partial_{t}^{2}-\Delta_{x}\right) u(x, t)=0$, $u(x, 0)=0,\left(\partial_{t} u\right)(x, 0)=f(x)$. We recall (see equation (5.4) in [5]) that

$$
k_{0}(x, t)=\pi(2 \pi)^{-n}(-1)^{m} \int_{\mathrm{S}^{n-1}} \delta^{(n-2)}(\langle x, \omega\rangle-t) \mathrm{d} \omega
$$

when $t \geq 0$.

LEMмA 1.3. We have the identity

$$
Q(f, g, h)=-4 \iint_{\mathrm{R}^{n} \times \mathrm{R}^{+}} A(f, g)(x, t)(\cos t|D| h)(x) \mathrm{d} x \mathrm{~d} t
$$

when $f, g, h \in C_{0}^{\infty}\left(\mathrm{R}^{n}\right)$.

Proof. By using the homogeneity of $E$ we get

$$
Q(f, g, h)=4 \iiint E(y, z) h(x-z) f(x+y) g(x-y) \mathrm{d} x \mathrm{~d} y \mathrm{~d} z .
$$

We recall that (see Thm. 10.4 of [5])

$$
E(y, z)=-\int_{0}^{\infty} k_{0}(y, t) \dot{k}_{0}(z, t) \mathrm{d} t .
$$

Then we first integrate with respect to $z$ in (1.7) and apply (1.8) to write

$$
\int E(y, z) h(x-z) \mathrm{d} z=-\int_{0}^{\infty} k_{0}(y, t)(\cos (t|D|) h)(x) \mathrm{d} t .
$$

Then the integration with respect to $y$ in (1.7) gives

$$
\int k_{0}(y, t) f(x+y) g(x-y) \mathrm{d} y=A(f, g)(x, t) .
$$

The formula (1.6) is then obtained by integrating over the remaining variables $x$ and $t$.

The main idea is to use continuity properties of the operators $\cos (t|D|)$ and $A$ in $H_{(a, b)}$ spaces in order to get the needed estimates. To this end we need an interpolation result for bilinear operators, which is contained in Section 2. Continuity properties of $\cos (t|D|)$ and $A$ are obtained in Section 3 , and the proof of the main result is then derived. 


\section{An interpolation result for bilinear operators}

In this subsection we consider general dimensions $d \geq 1$.

When $a, b \in \mathrm{R}$ we define

$$
H_{(a, b)}\left(\mathrm{R}^{d}\right)=\left\{u \in \mathscr{S}^{\prime}\left(\mathrm{R}^{d}\right) ;\langle x\rangle^{a}\langle D\rangle^{b} u \in L^{2}\left(\mathrm{R}^{d}\right)\right\} .
$$

This is a Hilbert space with norm

$$
\|u\|_{(a, b)}=\left\|\langle x\rangle^{a}\langle D\rangle^{b} u\right\|,
$$

where the norm in the right-hand side denotes the $L^{2}$ norm. Since the operators $\langle D\rangle^{b}\langle x\rangle^{a}\langle D\rangle^{-b}\langle x\rangle^{-a}$ and $\langle x\rangle^{a}\langle D\rangle^{b}\langle x\rangle^{-a}\langle D\rangle^{-b}$ are continuous in $L^{2}$, it follows that

$$
H_{(a, b)}\left(\mathrm{R}^{d}\right)=\left\{u \in \mathscr{S}^{\prime}\left(\mathrm{R}^{d}\right) ;\langle D\rangle^{b}\langle x\rangle^{a} u \in L^{2}\left(\mathrm{R}^{d}\right)\right\}
$$

and the norms $\|u\|_{(a, b)}$ and $\|u\|_{(a, b)}^{\prime}=\left\|\langle D\rangle^{b}\langle x\rangle^{a} u\right\|$ are equivalent. This in turn implies that the Fourier transform is a linear homeomorphism from $H_{(a, b)}$ onto $H_{(b, a)}$.

Assume $T: \mathscr{S}\left(\mathrm{R}^{d}\right) \times \mathscr{S}\left(\mathrm{R}^{d}\right) \rightarrow \mathscr{S}\left(\mathrm{R}^{N}\right)$ is a continuous bilinear operator. Let $I(T)$ be the set of all $\sigma=\left(a^{\prime}, b^{\prime}, a^{\prime \prime}, b^{\prime \prime}, a, b\right) \in \mathrm{R}^{6}$ for which there is a constant $C=C(\sigma)$ such that

$$
\|T(f, g)\|_{(a, b)} \leq C\|f\|_{\left(a^{\prime}, b^{\prime}\right)}\|g\|_{\left(a^{\prime \prime}, b^{\prime \prime}\right)}, \quad f, g \in \mathscr{S}\left(\mathrm{R}^{d}\right) .
$$

The next theorem might be obtained as an application of Theorem 4.4.1 in [2]. For the reader's convenience we include here a direct proof.

THEOREM 2.1. The set $I(T)$ is convex in $\mathrm{R}^{6}$.

We are going to use the following lemma.

Lemma 2.2. Assume $K \subset \mathrm{R}$ is a compact set. Then there is a positive constant $C$ depending on $K$ and $d$ only such that

$$
\left\|\langle D\rangle^{b}\langle x\rangle^{\mathrm{i} t}\langle D\rangle^{-b}\right\|_{L^{2}\left(\mathrm{R}^{d}\right) \rightarrow L^{2}\left(\mathrm{R}^{d}\right)} \leq(1+C|t|)^{|\operatorname{Re} b|},
$$

when $b \in \mathrm{C}$ with $\operatorname{Re} b \in K$ and $t \in \mathrm{R}$.

Proof. Choose a positive integer $M$ such that $K \subseteq[-2 M, 2 M]$ and set

$$
P_{M}(z, t)=\langle D\rangle^{2 M z}\langle x\rangle^{\mathrm{i} t}\langle D\rangle^{-2 M z}
$$

when $z \in \mathrm{C}, t \in \mathrm{R}$. We have that

$$
D_{j} \circ\langle x\rangle^{\mathrm{i} t}=\langle x\rangle^{\mathrm{i} t} \circ D_{j}+t x_{j}\langle x\rangle^{\mathrm{i} t-2} .
$$


It follows by induction over $|\alpha|$ that

$$
D^{\alpha} \circ\langle x\rangle^{\mathrm{i} t}=\langle x\rangle^{\mathrm{i} t} \circ D^{\alpha}+\sum_{\substack{1 \leq k \leq|\alpha| \\|\beta|<|\alpha|}}\langle x\rangle^{\mathrm{i} t} t^{k} p_{k, \alpha, \beta}(x) \circ D^{\beta},
$$

where $\langle x\rangle^{|\gamma|} \partial^{\gamma} p_{k, \alpha, \beta}$ is bounded for every $\gamma \in \mathbf{N}^{d}$. Hence there is a constant $C$, which depends on $M$ and $d$ only, such that

$$
\left\|P_{M}(1, t)\right\|_{L^{2} \rightarrow L^{2}} \leq(1+C|t|)^{2 M} .
$$

Since $\langle D\rangle^{2 M \mathrm{I} \operatorname{Im} z}$ is unitary in $L^{2}$ it follows that

$$
\left\|P_{M}(z, t)\right\|_{L^{2} \rightarrow L^{2}} \leq(1+C|t|)^{2 M} \quad \text { when } \quad \operatorname{Re} z=1, t \in \mathrm{R} .
$$

One also clearly has that

$$
\left\|P_{M}(z, t)\right\|_{L^{2} \rightarrow L^{2}} \leq 1 \quad \text { when } \quad \operatorname{Re} z=0, t \in \mathrm{R} .
$$

Let $f, g \in \mathscr{S}\left(\mathrm{R}^{d}\right)$ satisfy $\|f\|=\|g\|=1$ and set

$$
q_{M}(z, t)=(1+C|t|)^{-2 z M}\left\langle P_{M}(z, t) f, g\right\rangle .
$$

This is an entire analytic function, bounded on the strip $0 \leq \operatorname{Re} z \leq 1$ and, by (2.4) and (2.5), $\left|q_{M}(z, t)\right| \leq 1$ when $\operatorname{Re} z=0$ or $\operatorname{Re} z=1$. It follows by the three lines theorem that $\left|q_{M}(z, t)\right| \leq 1$ when $0 \leq \operatorname{Re} z \leq 1$. This implies that

$$
\left\|P_{M}(z, t)\right\|_{L^{2} \rightarrow L^{2}} \leq(1+C|t|)^{2 M|\operatorname{Re} z|}
$$

when $0 \leq \operatorname{Re} z \leq 1, t \in \mathrm{R}$. A similar proof shows that the above inequality holds also for $-1 \leq \operatorname{Re} z \leq 0$. The lemma follows after replacing $z$ by $b /(2 M)$.

Proof of Theorem 2.1. Assume

$$
\sigma_{0}=\left(a_{0}^{\prime}, b_{0}^{\prime}, a_{0}^{\prime \prime}, b_{0}^{\prime \prime}, a_{0}, b_{0}\right), \quad \sigma_{1}=\left(a_{1}^{\prime}, b_{1}^{\prime}, a_{1}^{\prime \prime}, b_{1}^{\prime \prime}, a_{1}, b_{1}\right)
$$

are elements of $I(T)$. Define

$$
\sigma(z)=\left(a^{\prime}(z), b^{\prime}(z), a^{\prime \prime}(z), b^{\prime \prime}(z), a(z), b(z)\right)=(1-z) \sigma_{0}+z \sigma_{1}, \quad z \in \mathrm{C} .
$$

Let $f, g \in \mathscr{S}\left(\mathrm{R}_{x}^{d}\right)$ and $h \in \mathscr{S}\left(\mathrm{R}_{y}^{N}\right)$ and set

$$
F(z)=\langle x\rangle^{-a^{\prime}(z)}\langle D\rangle^{-b^{\prime}(z)} f, \quad G(z)=\langle x\rangle^{-a^{\prime \prime}(z)}\langle D\rangle^{-b^{\prime \prime}(z)} g
$$

and

$$
H(z)=\langle y\rangle^{a(z)}\langle D\rangle^{b(z)} h .
$$


Then $F, G$ and $H$ are holomorphic functions of $z$ with values in $\mathscr{S}\left(\mathrm{R}^{d}\right)$ and $\mathscr{S}\left(\mathrm{R}^{N}\right)$, respectively, and their $\mathscr{S}$ seminorms have at most polynomial growth in $|z|$ when $\operatorname{Re} z$ stays in a bounded set.

The previous lemma shows that when $\operatorname{Re} z=0$

$$
\begin{aligned}
\|F(z)\|_{\left(a_{0}^{\prime}, b_{0}^{\prime}\right)} & \leq C_{1}\left\|\langle D\rangle^{b_{0}^{\prime}}\langle x\rangle^{z\left(a_{0}^{\prime}-a_{1}^{\prime}\right)}\langle D\rangle^{-b_{0}^{\prime}}\langle D\rangle^{z\left(b_{0}^{\prime}-b_{1}^{\prime}\right)} f\right\| \\
& \leq C_{2}(1+|\operatorname{Im} z|)^{\left|b_{0}^{\prime}\right|}\left\|\langle D\rangle^{z\left(b_{0}^{\prime}-b_{1}^{\prime}\right)} f\right\| \\
& \leq C|1+z|^{\left|b_{0}^{\prime}\right|}\|f\| .
\end{aligned}
$$

Similarly one gets

$$
\|G(z)\|_{\left(a_{0}^{\prime \prime}, b_{0}^{\prime \prime}\right)} \leq C|1+z|^{\left|b_{0}^{\prime \prime}\right|}\|g\|, \quad\|H(z)\|_{\left(-a_{0},-b_{0}\right)} \leq C|1+z|^{\left|b_{0}\right|}\|h\|
$$

when $\operatorname{Re} z=0$, and

$$
\begin{aligned}
\|F(z)\|_{\left(a_{1}^{\prime}, b_{1}^{\prime}\right)} & \leq C|1+z|^{\left|b_{1}^{\prime}\right|}\|f\|, \\
\|G(z)\|_{\left(a_{1}^{\prime \prime}, b_{1}^{\prime \prime}\right)} & \leq C|1+z|^{\left|b_{1}^{\prime \prime}\right|}\|g\|, \\
\|H(z)\|_{\left(-a_{1},-b_{1}\right)} & \leq C|1+z|^{\left|b_{1}\right|}\|h\|
\end{aligned}
$$

when $\operatorname{Re} z=1$.

Define

$$
q(z)=\langle T(F(z), G(z)), H(z)\rangle .
$$

This is an entire analytic function.

Since $T$ is continuous from $\mathscr{S}\left(\mathrm{R}^{d}\right) \times \mathscr{S}\left(\mathrm{R}^{d}\right)$ to $\mathscr{S}\left(\mathrm{R}^{N}\right)$ it follows (by using commutator estimates as in the previous lemma) that $q(z)$ is of at most polynomial growth in the strip $0 \leq \operatorname{Re} z \leq 1$. Since $\sigma_{0}, \sigma_{1} \in I(T)$ the estimates for $F, G, H$ above show that there are positive constants $C$ and $\gamma$, which are independent of $f, g, h$, such that $\left|(1+z)^{-\gamma} q(z)\right| \leq C\|f\| \cdot\|g\| \cdot\|h\|$ when $\operatorname{Re} z=0$ or $\operatorname{Re} z=1$. It follows then from the three lines theorem that $(1+z)^{-\gamma} q(z)$ satisfies the same estimate for every $z$ in the whole strip. When $z=\theta \in(0,1)$ we get an estimate for $q(\theta)$, and hence the estimate

$$
\left\|\langle D\rangle^{b(\theta)}\langle y\rangle^{a(\theta)} T\left(\langle x\rangle^{-a^{\prime}(\theta)}\langle D\rangle^{-b^{\prime}(\theta)} f,\langle x\rangle^{-a^{\prime \prime}(\theta)}\langle D\rangle^{-b^{\prime \prime}(\theta)} g\right)\right\| \leq C\|f\|\|g\|,
$$

where $C$ is independent of $f$ and $g$. This means precisely that $\sigma(\theta) \in I(T)$.

\section{Proof of the main result}

We recall that $n \geq 3$ is odd and we have denoted $m=(n-3) / 2$. We define the operator $K: \mathscr{S}^{\prime}\left(\mathrm{R}^{n}\right) \rightarrow \mathscr{S}^{\prime}\left(\mathrm{R}^{n+1}\right)$ through

$$
(K u)(x, t)=Y_{+}(t) \cos (t|D|) u(x), \quad t \in \mathrm{R}, x \in \mathrm{R}^{n},
$$


where $Y_{+}$is the characteristic function of $[0, \infty)$.

Proposition 3.1. Assume $a<0$ and $b \leq 0$. Then the operator $K$ is continuous from $H_{(a, b)}\left(\mathrm{R}^{n}\right)$ to $H_{(a-1 / 2, b)}\left(\mathrm{R}^{n+1}\right)$.

Proof. When $t \geq 0$ we denote by $\Lambda_{t}$ the operator on $\mathscr{S}\left(\mathrm{R}^{n}\right)$ which is multiplication by the function $\left((1+t)^{2}+|x|^{2}\right)^{1 / 2}$ and we consider $K(t)=$ $\cos (t|D|)$ as an operator in $\mathscr{S}\left(\mathrm{R}^{n}\right)$. Since $n$ is odd the convolution kernel $\dot{k}_{0}(x, t)$ of $K(t)$ is supported in the set where $|x|=t$. Therefore $K(t) f$ is supported in the ball with centre $x_{0}$ and radius $r+t$ if $f \in C_{0}^{\infty}\left(\mathrm{R}^{n}\right)$ is supported in the ball with centre $x_{0}$ and radius $r$.

Assume first that $a$ is real, arbitrary. We prove that there exists a constant $C_{a}$ such that

$$
\left\|\Lambda_{t}^{-a} K(t) \Lambda_{t}^{a}\right\|_{L^{2}\left(\mathrm{R}^{n}\right) \rightarrow L^{2}\left(\mathrm{R}^{n}\right)} \leq C_{a}, \quad t \geq 0 .
$$

Let $\left(T_{\sigma}\right)_{\sigma>0}$ be the dilation group on $\mathscr{S}\left(\mathrm{R}^{n}\right)$ defined by $T_{\sigma} h(x)=\sigma^{n / 2} h(\sigma x)$. Then $T_{\sigma}^{-1}=T_{1 / \sigma}$ and $T_{\sigma}$ extends to a unitary operator in $L^{2}$ for every $\sigma$. We notice that

$$
T_{\sigma} K(t) T_{\sigma}^{-1}=K(t / \sigma)
$$

and

$$
T_{1+t} \Lambda_{t}^{a} T_{1+t}^{-1}=(1+t)^{a} \Lambda_{0}^{a} .
$$

It follows that

$$
\Lambda_{t}^{-a} K(t) \Lambda_{t}^{a}=T_{1+t}^{-1} \Lambda_{0}^{-a} K(t /(1+t)) \Lambda_{0}^{a} T_{1+t} .
$$

Therefore, it is enough to show that for $0 \leq t \leq 1$ the operator $\Lambda_{0}^{-a} K(t) \Lambda_{0}^{a}$ extends to a bounded operator on $L^{2}\left(\mathrm{R}^{n}\right)$ and that there exists $C_{a}>0$ such that

$$
\left\|\Lambda_{0}^{-a} K(t) \Lambda_{0}^{a}\right\|_{L^{2}\left(\mathrm{R}^{n}\right) \rightarrow L^{2}\left(\mathrm{R}^{n}\right)} \leq C_{a}, \quad 0 \leq t \leq 1 .
$$

Take $0 \leq t \leq 1$. We notice that $(K(t) f, K(t) g)=0$ if $\operatorname{dist}(\operatorname{supp}(f)$, $\operatorname{supp}(g))>2$, since the supports of $K(t) f$ and $K(t) g$ do not overlap. Let $0 \leq \chi \in C_{0}^{\infty}\left(\mathrm{R}^{n}\right)$ be supported in the unit ball and satisfy $\int \chi(y) \mathrm{d} y=1$. For $f \in C_{0}^{\infty}\left(\mathrm{R}^{n}\right)$ define $f_{y}(x)=f(x) \chi(x-y)$. Then

$$
\left(K(t) f_{y}, K(t) f_{z}\right)=0 \quad \text { when } \quad|y-z| \geq 4 .
$$


Since $f=\int f_{y} \mathrm{~d} y$ it follows that

$$
\begin{aligned}
\left(\Lambda_{0}^{-a}\right. & \left.K(t) \Lambda_{0}^{a} f, \Lambda_{0}^{-a} K(t) \Lambda_{0}^{a} f\right) \\
\quad & =\iint\left(\Lambda_{0}^{-a} K(t) \Lambda_{0}^{a} f_{y}, \Lambda_{0}^{-a} K(t) \Lambda_{0}^{a} f_{z}\right) \mathrm{d} y \mathrm{~d} z \\
\quad & \iint_{|y-z| \leq 4}\left(\Lambda_{0}^{-a} K(t) \Lambda_{0}^{a} f_{y}, \Lambda_{0}^{-a} K(t) \Lambda_{0}^{a} f_{z}\right) \mathrm{d} y \mathrm{~d} z \\
\quad \leq & C \int\left\|\Lambda_{0}^{-a} K(t) \Lambda_{0}^{a} f_{y}\right\|^{2} \mathrm{~d} y .
\end{aligned}
$$

Since $|x-y| \leq 1$ in the support of $\Lambda_{0}^{a} f_{y}$, we have that $|x-y| \leq t+1$ in the support of $K(t) \Lambda_{0}^{a} f_{y}$. Hence

$$
\left\|\Lambda_{0}^{-a} K(t) \Lambda_{0}^{a} f_{y}\right\| \leq C_{1}\langle y\rangle^{-a}\left\|\Lambda_{0}^{a} f_{y}\right\| \leq C\left\|f_{y}\right\| .
$$

The proof of (3.3) is then completed by the fact that

$$
\int\left\|f_{y}\right\|^{2} \mathrm{~d} y \leq C\|f\|^{2} .
$$

Using (3.2) we get, when $a<0$,

$$
\begin{aligned}
\iint & \left(1+|x|^{2}+t^{2}\right)^{a-1 / 2}|K u(x, t)|^{2} \mathrm{~d} x \mathrm{~d} t \\
& =\iint\left(1+|x|^{2}+t^{2}\right)^{a-1 / 2}|(K(t) u)(x)|^{2} \mathrm{~d} x \mathrm{~d} t \\
& \leq C \iint\left(1+|x|^{2}+t^{2}\right)^{a-1 / 2}|u(x)|^{2} \mathrm{~d} x \mathrm{~d} t \\
& =C\left(\int_{-\infty}^{\infty}\left(1+t^{2}\right)^{a-1 / 2} \mathrm{~d} t\right) \int\left(1+|x|^{2}\right)^{a}|u(x)|^{2} \mathrm{~d} x=C^{\prime}\|u\|_{(a, 0)}^{2}
\end{aligned}
$$

when $u \in \mathscr{S}\left(\mathrm{R}^{n}\right)$. This concludes the proof for the case $b=0$, since $\mathscr{S}\left(\mathrm{R}^{n}\right)$ is dense in $H_{(a, 0)}$.

In the case $b<0$ the proposition follows from the fact that $K$ commutes with $D_{x}$ and the operator $\left(1+\left|D_{t}\right|^{2}+\left|D_{x}\right|^{2}\right)^{b}\left\langle D_{x}\right\rangle^{-b}$ is bounded. lary.

The previous proposition combined with Lemma 1.3 gives the next corol-

Corollary 3.2. Assume $a_{1}, a_{2}, b_{1}, b_{2}, a_{3}, b_{3} \in \mathrm{R}, a_{3}>0$ and $b_{3} \geq 0$. Then $B_{2}$ is continuous from $H_{\left(a_{1}, b_{1}\right)} \times H_{\left(a_{2}, b_{2}\right)}$ to $H_{\left(a_{3}, b_{3}\right)}$ if $A$ is continuous from $H_{\left(a_{1}, b_{1}\right)} \times H_{\left(a_{2}, b_{2}\right)}$ to $H_{\left(a_{3}+1 / 2, b_{3}\right)}$. 
We turn our attention to proving continuity properties for the bilinear operator $A$. We will first establish some useful formulas for $A(f, g)$ and its Fourier transform.

Let $S: \mathscr{S}\left(\mathrm{R}^{n}\right) \times \mathscr{S}\left(\mathrm{R}^{n}\right) \rightarrow \mathscr{S}\left(\mathrm{R}^{n+1}\right)$ be the operator defined through

$$
S(f, g)(x, t)=t^{m+1} \int_{S^{n-1}} f(x+t \omega) g(x-t \omega) \mathrm{d} \omega, \quad x \in \mathrm{R}^{n}, t \in \mathrm{R} .
$$

It is easy to see that $S$ extends to a bounded operator from $L^{2}\left(\mathrm{R}^{n}\right) \times L^{2}\left(\mathrm{R}^{n}\right)$ to $L^{2}\left(\mathrm{R}^{n+1}\right)$.

Lemma 3.3. Let $\widehat{A}(f, g)(\xi, \tau)$ denote the Fourier transform of $A(f, g)$ with respect to both variables. Then

$$
\widehat{A}(f, g)(\xi, \tau)=\frac{(\tau / 2)^{m}}{2^{3} i(2 \pi)^{n-1}} S(\hat{f}, \hat{g})(\xi / 2, \tau / 2),
$$

when $f, g \in \mathscr{S}\left(\mathrm{R}^{n}\right)$.

Proof. Let $\phi(\xi, t)$ be the Fourier transform of $A(f, g)(x, t)$ in the variable $x$. Then

$$
\begin{aligned}
\phi(\xi, t) & =(2 \pi)^{-n} \iint k_{0}(y, t) \widehat{f}(\eta) \widehat{g}(\xi-\eta) \mathrm{e}^{-\mathrm{i}\langle 2 \eta-\xi, y\rangle} \mathrm{d} y \mathrm{~d} \eta \\
& =(2 \pi)^{-n} 2^{-n} \iint k_{0}(y, t) \widehat{f}\left(\frac{\xi+\eta}{2}\right) \widehat{g}\left(\frac{\xi-\eta}{2}\right) \mathrm{e}^{-\mathrm{i}\langle\eta, y\rangle} \mathrm{d} y \mathrm{~d} \eta \\
& =(2 \pi)^{-n} 2^{-n} \int \frac{\sin (|\eta| t)}{|\eta|} \widehat{f}\left(\frac{\xi+\eta}{2}\right) \widehat{g}\left(\frac{\xi-\eta}{2}\right) \mathrm{d} \eta .
\end{aligned}
$$

It follows that

$$
\begin{aligned}
\widehat{A}(f, g)(\xi, \tau) & \\
= & (2 \pi)^{-n} 2^{-n} \iint_{\mathrm{R}^{n} \times \mathrm{R}} \mathrm{e}^{-\mathrm{i} \tau t} \frac{\mathrm{e}^{\mathrm{i} t|\eta|}-\mathrm{e}^{-\mathrm{i} t|\eta|}}{2 \mathrm{i}|\eta|} \widehat{f}\left(\frac{\xi+\eta}{2}\right) \widehat{g}\left(\frac{\xi-\eta}{2}\right) \mathrm{d} t \mathrm{~d} \eta \\
= & (2 \pi)^{-(n-1)} \mathrm{i}^{-1} 2^{-(n+1)}|\tau|^{-1} \\
& \quad \int_{\mathrm{R}^{n}}(\delta(|\eta|-\tau)-\delta(|\eta|+\tau)) \widehat{f}\left(\frac{\xi+\eta}{2}\right) \widehat{g}\left(\frac{\xi-\eta}{2}\right) \mathrm{d} \eta \\
= & (2 \pi)^{-(n-1)} \mathrm{i}^{-1} 2^{-(n+1)} \tau^{n-2} \int_{\mathrm{s}^{n-1}} \widehat{f}\left(\frac{\xi+\tau \omega}{2}\right) \widehat{g}\left(\frac{\xi-\tau \omega}{2}\right) \mathrm{d} \omega .
\end{aligned}
$$


This combined with (3.4) gives the lemma.

Lemma 3.4. We have

$$
k_{0}(x, t)=\partial_{t}^{m} \kappa_{0}(x, t),
$$

where the smooth mapping $\mathrm{R} \ni t \rightarrow \kappa_{0}(\cdot, t) \in \mathscr{D}^{\prime}\left(\mathrm{R}^{n}\right)$ is given by

$$
\begin{aligned}
\left\langle\kappa_{0}(\cdot, t), \varphi\right\rangle=\pi(2 \pi)^{-(n+1) / 2} t^{m+1} \int_{S^{n-1}} \varphi(t \omega) \mathrm{d} \omega \\
+\int_{t}^{\infty} p(t / r) r^{m}\left(\int_{S^{n-1}} \varphi(r \omega) \mathrm{d} \omega\right) \mathrm{d} r
\end{aligned}
$$

for every $\varphi \in C_{0}^{\infty}\left(\mathrm{R}^{n}\right)$. Here

$$
p(s)=\frac{1}{m !(4 \pi)^{m+1}}\left(-\frac{\mathrm{d}}{\mathrm{d} s}\right)^{m+1}\left(1-s^{2}\right)^{m},
$$

with the convention that $p(s)=0$ when $m=0$.

Remark 3.5. When $m=0$, that is, $n=3$ the polynomial $p$ vanishes. Therefore only the first term appears in the right-hand side of (3.7). We have used here the convention that $0 !=1$.

Proof. We notice that $p(s)$ is a polynomial of degree $m-1$ which is odd (even) if $m$ is even (odd). The polynomial $r^{m} p(t / r)$ is therefore odd in $r$ and odd (even) in $t$ if $m$ is even (odd). Set $\widetilde{\varphi}(t)=\int_{s^{n-1}} \varphi(t \omega) \mathrm{d} \omega$ when $t \in \mathrm{R}$. This is a smooth and even function of $t$. If $m$ is even then

$$
\int_{t}^{\infty} p(t / r) r^{m} \widetilde{\varphi}(r) \mathrm{d} r=\int_{-t}^{\infty} p(t / r) r^{m} \widetilde{\varphi}(r) \mathrm{d} r=-\int_{-t}^{\infty} p(-t / r) r^{m} \widetilde{\varphi}(r) \mathrm{d} r
$$

which shows that the left-hand side is an odd function of $t$. If $m$ is odd similar arguments show that the left-hand side is even in $t$. Hence, if we define $\kappa_{0}$ as in the lemma it follows that $\kappa_{0}(\cdot, t)$ is a smooth distribution valued function of $t$ which is odd (even) if $m$ is even (odd).

Define

$$
U_{0}(x, t)=\int_{\mathrm{S}^{n-1}} \delta^{(m+1)}(\langle x, \omega\rangle-t) \mathrm{d} \omega .
$$

It follows from (1.5) that

$$
k_{0}(x, t)=\partial_{t}^{m} \pi(2 \pi)^{-n} U_{0}(x, t) .
$$


Here $U_{0}(\cdot, t)$ is a smooth distribution valued function of $t$ with the same parity as $\kappa_{0}(\cdot, t)$. The lemma follows therefore if we prove that

$$
\kappa_{0}(x, t)=\pi(2 \pi)^{-n} U_{0}(x, t)
$$

when $t>0$.

We may write

$$
\begin{aligned}
U_{0}(x, t) & =\left(-\partial_{t}\right)^{m+2} \int_{s^{n-1}} Y_{+}(\langle x, \omega\rangle-t) \mathrm{d} \omega \\
& =c_{n-1}\left(-\partial_{t}\right)^{m+2} \int_{-1}^{1} Y_{+}(|x| s-t)\left(1-s^{2}\right)^{m} \mathrm{~d} s \\
& =c_{n-1}\left(-\partial_{t}\right)^{m+1} \int_{-1}^{1} \delta(|x| s-t)\left(1-s^{2}\right)^{m} \mathrm{~d} s,
\end{aligned}
$$

where $c_{n-1}=2 \pi^{m+1} / m$ ! is the area of the $(n-2)$-dimensional unit sphere. In $\{t>0\}$ we have

$$
\begin{aligned}
\left\langle U_{0}(\cdot, t), \varphi\right\rangle & =c_{n-1}\left(-\partial_{t}\right)^{m+1} \int_{0}^{\infty} \int_{-1}^{1} \delta(s r-t) r^{n-1}\left(1-s^{2}\right)^{m} \tilde{\varphi}(r) \mathrm{d} s \mathrm{~d} r \\
& =c_{n-1}\left(-\partial_{t}\right)^{m+1} \int_{0}^{1}(t / s)^{n-1}\left(1-s^{2}\right)^{m} s^{-1} \tilde{\varphi}(t / s) \mathrm{d} s \\
& =c_{n-1}\left(-\partial_{t}\right)^{m+1} \int_{t}^{\infty} r^{n-2}\left(1-t^{2} / r^{2}\right)^{m} \tilde{\varphi}(r) \mathrm{d} r .
\end{aligned}
$$

Set $q(s)=c_{n-1}(-\mathrm{d} / \mathrm{d} s)^{m+1}\left(1-s^{2}\right)^{m}=\pi^{-1}(2 \pi)^{n} p(s)$. A simple computation then gives

$$
\begin{aligned}
\left\langle U_{0}(\cdot, t), \varphi\right\rangle & =c_{n-1} m ! 2^{m} t^{m+1} \widetilde{\varphi}(t)+\int_{t}^{\infty} q(t / r) r^{m} \widetilde{\varphi}(r) \mathrm{d} r \\
& =\pi^{-1}(2 \pi)^{n}\left\langle\kappa_{0}(\cdot t), \varphi\right\rangle .
\end{aligned}
$$

This finishes the proof of the lemma.

Corollary 3.6. With the notation in the previous lemma, we have

$$
\begin{aligned}
& A(f, g)(x, t) \\
& \quad=\partial_{t}^{m}\left(\pi(2 \pi)^{-(n+1) / 2} S(f, g)(x, t)+\int_{t}^{\infty} p(t / r) r^{-1} S(f, g)(x, r) \mathrm{d} r\right)
\end{aligned}
$$

for every $f, g \in C_{0}^{\infty}\left(\mathrm{R}^{n}\right)$. 
It has become clear that, in order to get continuity properties of $A$, we need to study the bilinear operator $S$. We start with an elementary lemma, where meas $(\cdot)$ denotes the surface measure on $\mathrm{S}^{n-1}$.

Lemma 3.7. There is a constant $C$ such that

(3.8) meas $\left(\left\{\omega \in \mathrm{S}^{n-1} ; r / 2<|x-t \omega|<2 r,|x+t \omega|<s\right\}\right) \leq C\left(\frac{s}{r}\right)^{n-1}$ when $r, s>0, x \in \mathrm{R}^{n}, t \in \mathrm{R}$.

Proof. It is enough to prove the lemma for $s<r / 4$. Denote

$$
M(x, t ; r, s)=\left\{\omega \in \mathrm{S}^{n-1} ; r / 2<|x-t \omega|<2 r,|x+t \omega|<s\right\} .
$$

Since

$$
\operatorname{meas}(M(x,-t ; r, s))=\operatorname{meas}(M(x, t ; r, s))
$$

we may assume $t \geq 0$.

If $\omega \in M(x, t ; r, s)$ we must have $\langle x, \omega\rangle \leq 0$. It follows that $r / 2<|x|+t<$ $2 \sqrt{2} r$ when $M(x, t ; r, s) \neq \emptyset$. Also ||$x|-t|<s<r / 4$, hence $|x|, t$ and $r$ are of the same order of magnitude. Using the fact that the push-forward of the measure $\mathrm{d} \omega$ on $\mathrm{S}^{n-1}$ under the mapping $\omega \mapsto \tau=\langle x, \omega\rangle /|x| \in[-1,1]$ is a multiple of the measure $\left(1-\tau^{2}\right)^{m} \mathrm{~d} \tau$ we easily see that

$$
\operatorname{meas}(M(x, t ; r, s)) \leq C \int_{N(x, t, s)}(1-\tau)^{m} \mathrm{~d} \tau,
$$

where

$$
N(x, t, s)=\left\{\tau \in(0,1) ;|x|^{2}+t^{2}-2|x| t \tau<s^{2}\right\} .
$$

Since

$$
\int_{N(x, t, s)}(1-\tau)^{m} \mathrm{~d} \tau \leq \int_{0}^{\frac{s^{2}-(|x|-t)^{2}}{2|x| t}} \tau^{m} \mathrm{~d} \tau \leq C\left(\frac{s^{2}-(|x|-t)^{2}}{2|x| t}\right)^{m+1},
$$

we have proved that

$$
\operatorname{meas}(M(x, t ; r, s)) \leq C\left(s^{2} /(|x| t)\right)^{\frac{n-1}{2}} .
$$

Recalling that $|x|$ and $t$ are of the same order of magnitude as $r$, we see that (3.8) holds.

Lemma 3.8. Assume $r, s>0, \phi, \psi \in C_{0}^{\infty}\left(\mathrm{R}^{n}\right), \phi$ is supported in the set where $r / 2<|x|<2 r, \psi$ is supported in the set where $|x|<s$, and $a \in \mathbf{R}$. Then there is a constant $C=C(a)$, independent of $r, s, \phi$ and $\psi$, such that

$$
\|S(\phi, \psi)\|_{(a, 0)} \leq C(s / r)^{m+1} \max \left(\langle r\rangle^{a},\langle r+s\rangle^{a}\right)\|\phi\|\|\psi\| .
$$


Proof. It follows from Lemma 3.7 and Cauchy's inequality applied to the integration with respect to $\omega$ that there is a constant $C$ such that (3.10)

$$
|S(\phi, \psi)(x, t)|^{2} \leq C t^{n-1}(s / r)^{2(m+1)} \int|\phi(x+t \omega)|^{2}|\psi(x-t \omega)|^{2} \mathrm{~d} \omega .
$$

Since $2\left(|x|^{2}+t^{2}\right)=|x+t \omega|^{2}+|x-t \omega|^{2}$ when $\omega \in S^{n-1}$, one has

$$
\begin{aligned}
& \quad\left(1+|x|^{2}+t^{2}\right)^{a}|S(\phi, \psi)(x, t)|^{2} \\
& \leq C t^{n-1}\left(\frac{s}{r}\right)^{2(m+1)} \int\left(1+|x+t \omega|^{2}+|x-t \omega|^{2}\right)^{a}|\phi(x+t \omega)|^{2}|\psi(x-t \omega)|^{2} \mathrm{~d} \omega \\
& \leq C_{1} t^{n-1}\left(\frac{s}{r}\right)^{2(m+1)} \max \left(\langle r\rangle^{2 a},\langle r+s\rangle^{2 a}\right) \int|\phi(x+t \omega)|^{2}|\psi(x-t \omega)|^{2} \mathrm{~d} \omega .
\end{aligned}
$$

An integration with respect to $x$ and $t$ in (3.11) gives (3.9).

Lemma 3.9. Let $a^{\prime}, a^{\prime \prime}, a \in \mathrm{R}$ satisfy

$$
a<m+1+\min \left(a^{\prime}, a^{\prime \prime}\right), \quad a \leq a^{\prime}+a^{\prime \prime} .
$$

Then $S$ is continuous from $H_{\left(a^{\prime}, 0\right)} \times H_{\left(a^{\prime \prime}, 0\right)}$ to $H_{(a, 0)}$.

Proof. Choose $\chi \in C_{0}^{\infty}\left(\mathrm{R}^{n}\right)$ a smooth decreasing function of $|x|$ such that $\chi(x)=1$ when $|x|<1, \chi(x)=0$ when $|x|>2$ and $0 \leq \chi \leq 1$. Set

$$
\chi_{j}(x)=\chi\left(2^{-j} x\right)-\chi\left(2^{1-j} x\right), \quad j \geq 1, \quad \chi_{0}(x)=\chi(x)
$$

when $x \in \mathrm{R}^{n}$. Then $f=\sum_{0}^{\infty} \chi_{j} f$ with convergence in $\mathscr{S}\left(\mathrm{R}^{n}\right)$ when $f$ is in that space. In addition, when $\rho \in \mathrm{R}$, there is $C=C(\rho) \geq 0$ such that

$$
C^{-1} \sum_{0}^{\infty} 2^{2 \rho j}\left\|\chi_{j} f\right\|^{2} \leq\|f\|_{(\rho, 0)}^{2} \leq C \sum_{0}^{\infty} 2^{2 \rho j}\left\|\chi_{j} f\right\|^{2} .
$$

Consider $f, g \in \mathscr{S}\left(\mathrm{R}^{n}\right)$ and denote $s_{j}=2^{a^{\prime} j}\left\|\chi_{j} f\right\|, \sigma_{k}=2^{a^{\prime \prime} k}\left\|\chi_{k} g\right\|$. These are $\ell^{2}(\mathrm{~N})$-sequences with $\ell^{2}$ norm bounded from above by a constant $C$ times $\|f\|_{\left(a^{\prime}, 0\right)}$ and $\|g\|_{\left(a^{\prime \prime}, 0\right)}$, respectively. Set $\varepsilon=m+1+\min \left(a^{\prime}, a^{\prime \prime}\right)-a$. Then $\varepsilon>0$ and we shall show that there is a constant $C>0$, which depends on $a, a^{\prime}, a^{\prime \prime}$ only, such that

$$
\left\|S\left(\chi_{j} f, \chi_{k} g\right)\right\|_{(a, 0)} \leq C 2^{-\varepsilon|j-k|} s_{j} \sigma_{k} .
$$


Hence

$$
\begin{aligned}
\|S(f, g)\|_{(a, 0)} & \leq \sum_{j, k \geq 0}\left\|S\left(\chi_{j} f, \chi_{k} g\right)\right\|_{(a, 0)} \leq C_{1} \sum_{j, k \geq 0} 2^{-\varepsilon|j-k|} s_{j} \sigma_{k} \\
& \leq C_{2}\left(\sum_{j \geq 0} s_{j}^{2}\right)^{1 / 2}\left(\sum_{j \geq 0} \sigma_{k}^{2}\right)^{1 / 2} \leq C\|f\|_{\left(a^{\prime}, 0\right)}\|g\|_{\left(a^{\prime \prime}, 0\right)} .
\end{aligned}
$$

This would prove the statement.

It remains to prove (3.14). Since $S$ is symmetric, and since the condition (3.12) is symmetric in $\left(a^{\prime}, a^{\prime \prime}\right)$, it suffices to prove (3.14) when $j \geq k$. The previous lemma shows that

$$
\left|S\left(\chi_{j} f, \chi_{k} g\right)\right| \leq C 2^{-\rho_{j k}} s_{j} \sigma_{k},
$$

where

$$
\begin{aligned}
\rho_{j k} & =(j-k)(m+1)-a j+a^{\prime} j+a^{\prime \prime} k \\
& =(j-k)\left(m+1+a^{\prime}-a\right)+\left(a^{\prime}+a^{\prime \prime}-a\right) k \\
& \geq(j-k)\left(m+1+\min \left(a^{\prime}, a^{\prime \prime}\right)-a\right) \\
& =(j-k) \varepsilon .
\end{aligned}
$$

This proves (3.14).

Lemma 3.10. Define

$$
T(f, g)(x, t)=\int_{t}^{\infty} p(t / r) r^{-1} S(f, g)(x, r) \mathrm{d} r,
$$

when $f, g \in C_{0}^{\infty}\left(\mathrm{R}^{n}\right)$. Then

$$
\|T(f, g)\|_{(a, 0)} \leq 2 \max _{|s| \leq 1}|p(s)| \cdot\|S(f, g)\|_{(a, 0)}
$$

when $a \geq 0$.

Proof. We recall that

$$
p(t / r) r^{-1} S(f, g)(x, r)=p(t / r) r^{m} r^{-(m+1)} S(f, g)(x, r)
$$

is an odd function of $r$. Hence

$$
\begin{aligned}
|T(f, g)(x, t)| & \leq\left|\int_{|t|}^{\infty} p(t / r) r^{-1} S(f, g)(x, r) \mathrm{d} r\right| \\
& \leq C \int_{|t|}^{\infty} r^{-1}|S(f, g)(x, r)| \mathrm{d} r
\end{aligned}
$$


where $C=\max _{|s| \leq 1}|p(s)|$. When $a \geq 0$ we get

$$
\left(1+|x|^{2}+t^{2}\right)^{a / 2}|T(f, g)(x, t)| \leq C \int_{|t|}^{\infty} r^{-1}\left(1+|x|^{2}+r^{2}\right)^{a / 2}|S(f, g)(x, r)| \mathrm{d} r .
$$

The lemma follows therefore if we notice that

$$
\int_{0}^{\infty} H^{2}(t) \mathrm{d} t \leq 4 \int_{0}^{\infty} h^{2}(t) \mathrm{d} t
$$

when $H(t)=\int_{t}^{\infty} t^{-1} h(t) \mathrm{d} t$ and $0 \leq h \in C_{0}(\mathrm{R})$. In fact, if $\widetilde{h}(s)=e^{s / 2} h\left(e^{s}\right)$ and $\widetilde{H}(s)=e^{s / 2} H\left(e^{s}\right)$, then

$$
\int_{0}^{\infty} h^{2}(t) \mathrm{d} t=\|\tilde{h}\|_{L^{2}(\mathrm{R})}^{2}, \quad \int_{0}^{\infty} H^{2}(t) \mathrm{d} t=\|\tilde{H}\|_{L^{2}(\mathrm{R})}^{2},
$$

and $\widetilde{H}=\gamma * \widetilde{h}$, where $\gamma(s)=\left(1-Y_{+}(s)\right) e^{s / 2}$ has $L^{1}$ norm equal to 2 .

Proposition 3.11. (i) When $a^{\prime}, a^{\prime \prime}, a \in \mathrm{R}$ satisfy

$$
0 \leq a<m+1+\min \left(a^{\prime}, a^{\prime \prime}\right), \quad a \leq a^{\prime}+a^{\prime \prime},
$$

then $A$ extends to a continuous bilinear operator from $H_{\left(a^{\prime}, 0\right)} \times H_{\left(a^{\prime \prime}, 0\right)}$ to $H_{(a,-m))}$.

(ii) When $b^{\prime}, b^{\prime \prime}, b \in \mathrm{R}$ satisfy

$$
b<m+1+\min \left(b^{\prime}, b^{\prime \prime}\right), \quad b \leq b^{\prime}+b^{\prime \prime},
$$

then $A$ extends to a continuous bilinear operator from $H_{\left(0, b^{\prime}\right)} \times H_{\left(0, b^{\prime \prime}\right)}$ to $H_{(0, b-m)}$.

Proof. A combination of Corollary 3.6, Lemma 3.9 and Lemma 3.10 gives (i), and (ii) follows from Lemma 3.3 and Lemma 3.9.

Proposition 3.12. Let $\left(a^{\prime}, b^{\prime}, a^{\prime \prime}, b^{\prime \prime}, a, b\right) \in \mathrm{R}^{6}$ satisfy

$$
\begin{gathered}
0 \leq a<m+1+\min \left(a^{\prime}, a^{\prime \prime}\right), \quad a \leq a^{\prime}+a^{\prime \prime}, \\
b<m+1+\min \left(b^{\prime}, b^{\prime \prime}\right), \quad b \leq b^{\prime}+b^{\prime \prime}, \\
a+b<m+1+\min \left(a^{\prime}, a^{\prime \prime}\right)+\min \left(b^{\prime}, b^{\prime \prime}\right) .
\end{gathered}
$$

Then $A$ is continuous from $H_{\left(a^{\prime}, b^{\prime}\right)} \times H_{\left(a^{\prime \prime}, b^{\prime \prime}\right)}$ to $H_{(a, b-m)}$.

Proof. If $\left(a^{\prime}, b^{\prime}, a^{\prime \prime}, b^{\prime \prime}, a, b\right) \in \mathrm{R}^{6}$ satisfies (3.16), it is easy to see that there is an $\theta \in(0,1)$ such that

$$
a<\theta(m+1)+\min \left(a^{\prime}, a^{\prime \prime}\right), \quad b<(1-\theta)(m+1)+\min \left(b^{\prime}, b^{\prime \prime}\right) .
$$


This shows that $a^{\prime} / \theta, a^{\prime \prime} / \theta, a / \theta$, respectively $b^{\prime} /(1-\theta), b^{\prime \prime} /(1-\theta), b /(1-\theta)$ satisfy the conditions in Proposition 3.11, hence

$$
\begin{aligned}
\left(a^{\prime} / \theta, 0, a^{\prime \prime} / \theta, 0, a / \theta,-m\right) & \in I(A), \\
\left(0, b^{\prime} /(1-\theta), 0, b^{\prime \prime} /(1-\theta), 0,-m+b /(1-\theta)\right) & \in I(A) .
\end{aligned}
$$

The proposition then follows by Theorem 2.1.

Proof of Theorem 1.1. Theorem 1.1 follows from Proposition 3.12 applied for $\left(a^{\prime}, b^{\prime}, a^{\prime \prime}, b^{\prime \prime}, a+1 / 2, b\right)$, and from Corollary 3.2.

\section{REFERENCES}

1. Beltiţă, I., and Melin, A., Local smoothing for the backscattering transform, preprint arXiv: 0712.3865 .

2. Bergh, J., and Löfström, J., Interpolation Spaces. An Introduction, Grundlehren der mathematischen Wissenschaften 223, Springer, Berlin, 1976.

3. Hörmander, L., The Analysis of Linear Partial Differential Operators I. Distribution Theory and Fourier Analysis, Grundlehren der mathematischen Wissenschaften 256, Springer, Berlin, 1983.

4. Melin, A., Smoothness of higher order terms in backscattering, in: Wave phenomena and asymptotic analysis, RIMS Kokyuroku 1315 (2003), 43-51.

5. Melin, A., Some transforms in potential scattering in odd dimension. pp. 103-134 in: Inverse Problems and Spectral Theory, Contemp. Math 348, Amer. Math. Soc., Providence, RI 2004.

6. Ruiz, A., Vargas, A., Partial recovery of a potential from backscattering data, Comm. Partial Differential Equations 30 (2005), 67-96.

INSTITUTE OF MATHEMATICS "SIMION STOILOW" OF THE ROMANIAN ACADEMY

PO BOX 1-764

BUCHAREST

ROMANIA

E-mail: Ingrid.Beltita@imar.ro
LUND UNIVERSITY

SWEDEN

E-mail: andersmelin@hotmail.com 